\title{
Les tableaux de William McTaggart (1835-1910), ou la société et l'histoire des Highlands selon un peintre de la côte ouest de l'Écosse
}

William McTaggart's Paintings, or Highland Society and History by a Painter from the West Coast of Scotland

\section{Marion Amblard}

\section{OpenEdition}

Journals

Édition électronique

URL : http://journals.openedition.org/etudesecossaises/775

DOI : 10.4000/etudesecossaises.775

ISSN : 1969-6337

Éditeur

UGA Éditions/Université Grenoble Alpes

Édition imprimée

Date de publication : 15 avril 2013

Pagination : 47-70

ISBN : 978-2-84310-246-2

ISSN : $1240-1439$

\section{Référence électronique}

Marion Amblard, « Les tableaux de William McTaggart (1835-1910), ou la société et l'histoire des Highlands selon un peintre de la côte ouest de l'Écosse », Études écossaises [En ligne], 16 | 2013, mis en ligne le 15 avril 2014, consulté le 16 mars 2021. URL : http://journals.openedition.org/ etudesecossaises/775; DOI : https://doi.org/10.4000/etudesecossaises.775 


\section{Les tableaux de William McTaggart (1835-1910), ou la société et l'histoire des Highlands selon un peintre de la côte ouest de l'Écosse}

Le 2 août 1918, le peintre Samuel John Peploe (1871-1935) écrivit à son ami et collègue Francis Campbell Boileau Cadell (1883-1937) qui se trouvait alors sur le front en France: "When the war is over I shall go to the Hebrides, recover some virtues I have lost. There is something marvellous about those western seas. Oh, Iona. We [Peploe, Cadell and Fergusson] must all go together next summer ${ }^{1}$.» Peploe, Cadell et John Duncan Fergusson (1874-1961) — trois des quatre peintres constituant le groupe d'artistes connu sous le nom des Colourists ${ }^{2}$ - allaient régulièrement peindre sur l'île d'Iona, dont ils appréciaient les paysages riches en couleurs et en contrastes accentués par la lumière qui règne sur l'île, alternant les ciels tantôt sereins tantôt tourmentés de nuages ${ }^{3}$. Les toiles de Cadell et Peploe représentant la mer turquoise et les plages de sable blanc d'Iona comptent désormais parmi les œuvres les plus célèbres de l'histoire de l'art pictural écossais. Les Colourists ne furent cependant pas les premiers artistes à peindre des vues des comtés du nord et de l'ouest de l'Écosse. Les peintres écossais se sont inspirés des Hautes Terres dès la fin du XVIII ${ }^{\mathrm{e}}$ siècle, lorsque des genres picturaux autres que le portrait

1. Cité dans G. Peploe, S. F. Peploe 1871-1935, Édimbourg et Londres, Mainstream publishing, 2000, p. 57.

2. Ce groupe de peintres comprenait aussi John Leslie Hunter (1877-1931). D'après Guy Peploe, petit-fils et biographe de Samuel John Peploe, il semblerait que Tom Honeyman ait été le premier à utiliser le terme «colourists» pour désigner Peploe, Cadell et Hunter. En 1950, il consacra à ces artistes un ouvrage intitulé Three Scottish Colourists. L'amitié qui liait ces trois peintres à Fergusson et l'attention particulière que ce dernier portait au traitement des couleurs expliquent que, désormais, Fergusson soit inclus dans le groupe des Colourists (ibid., p. 164).

3. En 1923, Peploe adressa une lettre à son ami William Macdonald dans laquelle il expliquait : "We had miserable weather in Iona this year-worst in living memory - gales and rain the whole time. $[\ldots]$ But that kind of weather suits Iona: the rocks and distant shores are seen through falling rain, veil behind veil, take on an elusive quality, and when the light shines through one had visions of rare beauty. I think I prefer it these days to your blue skies and clear distances. » (Cité dans ibid., p. 73.) 
ont commencé à se développer en Écosse. Auparavant, vers 1680, John Michael Wright (1617-1694) avait peint le portrait de Sir Mungo Murray vêtu d'un plaid, tenue traditionnelle des habitants des Highlands et, au début du XVIII ${ }^{\mathrm{e}}$ siècle, Richard Waitt (?-1732) exécuta une série de portraits des membres du clan Grant ainsi que les portraits de plusieurs chefs de clan ${ }^{4}$.

Alexander Runciman (1736-1785) et David Allan (1744-1796) furent les deux premiers peintres écossais à réaliser des toiles ayant pour sujet la culture et les coutumes des Highlands. À Penicuik, dans la demeure de son mécène Sir John Clerk, Runciman exécuta en 1772 des peintures murales représentant des scènes tirées des légendes d'Ossian, poème épique composé par James Macpherson (1736-1796) d'après d'anciennes légendes celtes ${ }^{5}$. Pour sa part, en 1780, Allan choisit de peindre une danse traditionnelle des Highlands dans une aquarelle intitulée The Highland Dance ${ }^{6}$. C'est aussi à Allan que l'on doit l'un des premiers paysages des Hautes Terres par un artiste écossais : il s'agit d'une vue du château de Blair et de la campagne environnante du comté de Perth qui constitue l'arrière-plan d'une conversation piece ${ }^{7}$ représentant le quatrième duc d'Atholl et sa famille.

4. Waitt peignit entre autres les portraits Alastair Mhor Grant, Champion of the Clan Grant (v. 1714, Glasgow Museums, Glasgow), The Hen Wife of Castle Grant (v. 1726, collection particulière), The Fool of the Laird of Grant (1731, National Portrait Gallery, Édimbourg) et The Grant Piper (v. 1714, National Museum of Scotland, Édimbourg). Andrew Macpherson of Cluny (v. 1725, National Galleries of Scotland, Édimbourg) et Kenneth Sutherland (v. 1712, National Galleries of Scotland, Édimbourg) sont deux des chefs de clan ayant posé pour Waitt. Par la suite, tout au long du XVIII ${ }^{\mathrm{e}}$ siècle, les portraitistes écossais, comme Allan Ramsay (1713-1784) et William Mosman (v. 1700-1771), peignirent régulièrement des tableaux représentant leurs modèles originaires des Hautes Terres vêtus d'un kilt.

5. Ces œuvres ont été détruites dans un incendie en 1899.

6. Allan s'intéressa aux coutumes des Highlands et réalisa notamment deux dessins consacrés aux danses et aux mariages traditionnels de la région. David Allan, A Highland Dance, D 5185 et A Highland Wedding, D 4411, cabinet des dessins et des estampes, National Gallery of Scotland, Édimbourg.

7. Les conversation pieces sont des tableaux représentant en pied des personnes occupées à des activités diverses et qui, comparés à des portraits individuels, étaient peints sur des toiles de format plus réduit. Ainsi que l'explique William Vaughan, ce genre pictural était particulièrement prisé en Grande-Bretagne au XVIII siècle et comprenait deux types de tableaux : "The "Conversation" was not a new kind of picture. What was new was the use of it for this particular kind of social portrait. [...] Throughout the eighteenth century a picture as a "Conversation" was as likely to be a fictional scene as it was to be a group portrait. [...] The earliest known reference to Conversations in Britain is to work by Heemskerk [sic], a producer of "lewd and bawdy scenes". [...] Low-life conversations remained a feature in the British scene. Hogarth, typically, painted many such Conversations.» (W. Vaughan, British painting. The golden age from Hogarth to Turner, Londres, Thames et Hudson, 1999, p. 51-52.)

En 1681, le peintre hollandais Jacob de Wet (1641-1697), qui travailla quelques années en Écosse, peignit le portrait de Patrick Lyon, $3^{\text {e }}$ comte de Strathmore et Kinghorne (Glamis Castle, Glamis), représentant en arrière-plan une vue des jardins et du château de Glamis. Il s'agit de l'un des premiers paysages représentant une vue des Highlands. 
La publication des légendes d'Ossian, des ouvrages de Sir Walter Scott (1771-1832), la définition d'une nouvelle identité écossaise pro-unioniste dont la spécificité reposait en partie sur l'assimilation de l'ensemble de l'Écosse aux Highlands ${ }^{8}$, ainsi que la visite officielle du roi Georges IV (1762-1830) en 1822 et les séjours de la reine Victoria (1819-1901) à Balmoral ont contribué à l'engouement collectif pour les Highlands alors que cette région traversait une crise profonde. De nombreux peintres surent profiter de ce vif intérêt pour les Hautes Terres : Horatio McCulloch (1805-1867), entre autres, se spécialisa dans la représentation des paysages des comtés du nord-ouest et le succès de ses toiles incita les paysagistes écossais à l'imiter pendant plus d'un demi-siècle ${ }^{9}$. Le peintre anglais Edwin Landseer (1802-1873) acquit quant à lui une renommée internationale grâce à ses tableaux représentant des parties de chasse dans les Highlands et des cerfs avec, en fond, un paysage montagneux. La représentation picturale des Hautes Terres et des habitants de cette région par des peintres écossais évolua pour les besoins de la nouvelle identité nationale définie au début du XIX ${ }^{\mathrm{e}}$ siècle et avec la manière dont les percevaient les Lowlanders. Ces derniers avaient longtemps méprisé les Highlanders qu'ils considéraient comme des ennemis et des opposants à l'Union des Parlements eu égard à leur soutien à la cause des Stuarts lors des soulèvements jacobites de 1715, 1719 et 1745; néanmoins, à l'issue des guerres napoléoniennes, en raison de leur engagement massif dans les troupes de l'armée britannique depuis la seconde moitié du XVIII siècle, les Highlanders furent perçus comme de fervents défenseurs de la GrandeBretagne et devinrent les représentants de l'Écosse et de la nouvelle

La représentation des paysages de l'Écosse et le développement de la peinture de paysage en Écosse ont été étudiés par Errington et Holloway. Voir L. Errington et J. Holloway, The Discovery of Scotland: the appreciation of Scottish scenery, Édimbourg, National Gallery of Scotland, 1978.

8. À l'issue des guerres napoléoniennes, Walter Scott, les hommes de lettres et les peintres ont défini une identité binationale à la fois écossaise et britannique, soulignant à la fois la spécificité culturelle de l'Écosse et son allégeance envers la Grande-Bretagne. Avec la révolution industrielle, les différences entre les Lowlands et l'Angleterre s'étaient amoindries; aussi, afin d'affirmer l'originalité culturelle de l'Écosse et assurer sa loyauté envers la Grande-Bretagne, Scott et les peintres écossais ont assimilé l'ensemble de l'Écosse aux Highlands.

Plusieurs ouvrages se sont intéressés à la définition de l’identité écossaise au début du XIXe siècle. Entre autres, Devine, Pittock et Womack sont revenus en détail sur le contexte dans lequel l'identité pro-unioniste fut définie. Voir T. M. Devine, The Scottish Nation 1700-2000; M. Pittock, Scottish Nationality, Basingstoke, Palgrave, 2001; T. C. Smout, A History of the Scottish people 1560-1830, Grande-Bretagne, Fontana, 1972.

9. Sur la carrière et l'œuvre de McCulloch, voir S. Smith, Horatio McCulloch 1805-1867, Glasgow, Glasgow Museums and Art Galleries, 1988. Sur les tableaux de McCulloch et la représentation des Highlands par les peintres écossais victoriens, voir M. Amblard, «Les tableaux de Horatio McCulloch et des paysagistes écossais du XIx siècle, ou l'envers du décor de l'identité écossaise prounioniste», Études écossaises, n 12, Grenoble, Ellug, 2009, p. 149-156. 
identité nationale ${ }^{10}$. C'est ainsi qu'en l'espace d'un siècle, la représentation picturale des Highlanders se transforma radicalement, comme en témoigne notamment la comparaison des portraits des membres du clan Grant par Waitt à celui de James Farquharson of Invercauld peint par Sir Francis Grant (1803-1878) en 1844'11. La représentation des paysages des Highlands évolua de manière comparable entre le XVIII ${ }^{\mathrm{e}}$ siècle et la première moitié du XIX ${ }^{e}$ siècle puisque les paysages, qui avaient horrifié les voyageurs qui s'étaient aventurés dans les Hautes Terres au XVIII siècle, suscitèrent une vive admiration dès le début du XIX ${ }^{\mathrm{e}}$ siècle et incitèrent un nombre croissant de touristes et de peintres à visiter la région. Parmi les nombreux tableaux que les artistes victoriens écossais ont consacrés aux Highlands, ceux de William McTaggart (1835-1910) se démarquent en raison de leur style et des sujets représentés.

Cet article a pour objectif de montrer qu'avec ses toiles, McTaggart propose une reconstitution de l'histoire des Highlands. Une étude de la peinture de paysage en Écosse durant la seconde moitié du XIX ${ }^{\mathrm{e}}$ siècle permet de constater que deux types de peintres et de tableaux se distinguent avec, d'une part, les artistes romantiques se spécialisant dans la représentation de paysages idéalisés des Highlands et, d'autre part, les artistes peignant des vues des Lowlands dans un style comparable à celui des écoles réaliste et naturaliste ${ }^{12}$ du continent. Le premier groupe de peintres comprend la majorité des paysagistes qui exercèrent à partir du début des années 1830 et dont les tableaux aidèrent à ancrer dans les esprits la synecdoque Highlands/Écosse et à diffuser la nouvelle identité

10. Ainsi que l'a souligné le professeur Tom Devine : «In subsequent eighteenth-century conflicts [from 1756 onwards] over fifty battalions of Highland troops were raised, distinguishing themselves at Quebec, Seringapatam, Waterloo and a host of other battles in many parts of the world. [...] It was not therefore surprising that the Highland regiments became a major factor in altering perceptions of Highland society after the '45. The speed of the transformation from Jacobite traitors to imperial heroes is astonishing. » (T. Devine, Clanship to Crofters' War. The transformation of the Scottish Highlands, p. 91-92.)

11. Pour plus de détails sur l'évolution de la représentation picturale des Highlanders, voir M. Amblard, «Du Rebelle au héros. Les Highlanders vus par les portraitistes des Lowlands entre 1680 et 1827 », Études écossaises, n 11, Grenoble, Ellug, 2008, p. 193-205.

12. Le terme réalisme désigne le mouvement pictural et littéraire développé entre les années 1840 et 1890. Dans son Vocabulaire d'esthétique, Étienne Souriau définit l'artiste réaliste comme étant «celui qui ne cherche pas l'évasion, le fictif, l'irréel, mais qui vise à faire passer dans son œuvre les êtres et les choses, tels qu'il les constate» (É. Souriau, «Réalisme», Vocabulaire d'esthétique, Paris, Quadrige et Presses universitaires de France, 2010, p. 1274). Selon É. Souriau, le naturalisme est un «mouvement artistique et littéraire, de la fin du Xix ${ }^{\mathrm{e}}$ siècle, rattaché à Zola qui en a formulé la théorie. Le naturalisme possède d'abord les caractères généraux du réalisme : peinture du réel sans en craindre les laideurs, intérêt pour la modernité psychologique et surtout sociale. Mais il y ajoute quelques différences spécifiques, qui en font une branche particulière du réalisme : rôle important accordé au physiologique dans les personnages, influence des théories scientifiques et particulièrement médicales $[\ldots] »(«$ Naturalisme», ibid., p. 1116). 
écossaise pro-unioniste. Ce ne fut qu'à partir de la fin des années 1860 que les paysages de style réaliste commencèrent à se multiplier, lorsqu'un nombre croissant de peintres commencèrent à critiquer les œuvres des paysagistes romantiques et à rejeter l'assimilation de l'ensemble de l'Écosse aux Highlands. Les toiles de McTaggart ne s'inscrivent dans aucune de ces deux catégories : s'il n'approuva pas le style des peintres romantiques et les clichés véhiculés par leurs tableaux, il ne se spécialisa pas pour autant dans la représentation des Lowlands. Il s'inspira de l'histoire des Highlands, des souvenirs de son enfance passée sur la côte ouest de l'Écosse et de ses nombreux séjours dans la région pour évoquer, à travers ses marines, les transformations subies par la société gaélique écossaise depuis le XVIII ${ }^{\mathrm{e}}$ siècle.

Il parait tout d'abord essentiel de retracer la carrière de William McTaggart et de replacer ses œuvres dans leur contexte culturel afin de mieux cerner ce qui distingue ses tableaux de ceux de ses contemporains écossais, et de comprendre les raisons pour lesquelles cet artiste occupe désormais une place majeure dans l'histoire de l'art pictural écossais. Une étude de ses tableaux évoquant l'arrivée de saint Colomba vers $565^{13}$ et de ses scènes d'exode inspirées de celles dont il avait été témoin pendant son enfance, nous permettra de constater que McTaggart revient sur trois événements marquants de l'histoire de la société gaélique : le début de la civilisation chrétienne en Écosse, les Clearances - qui furent à l'origine de l'émigration de plusieurs milliers d'habitants des Highlands aux XVIII ${ }^{\mathrm{e}}$ et $\mathrm{XIX}^{\mathrm{e}}$ siècles ${ }^{14}$ — et la fin des Clearances, symbolisée par le vote du Crofting Act en 1886. Ensemble, ces œuvres, dépourvues du sentimentalisme qui caractérise la plupart des tableaux de ses contemporains, offrent une vision de la société et de l'histoire des Highlands très différente de celle proposée par les peintres écossais depuis les années 1830.

William McTaggart naquit le 25 octobre 1835 dans un village de la côte ouest de l'Écosse situé sur la péninsule de Kintyre. Fils d'ouvriers agricoles, il grandit à Campbeltown; à douze ans, il devint l'assistant d'un apothicaire qui ne tarda pas à déceler son talent pour le dessin. Celui-ci, qui comptait parmi ses clients quelques notables de Campbeltown et des

13. Les spécialistes ne s'accordent pas sur la date exacte de la venue de saint Colomba en Écosse. Le professeur Christian Civardi situe l'arrivée du saint vers 565, tandis que Michel Duchein estime qu'il se serait installé sur l'île d'Iona vers 563. Voir C. Civardi, L'Écosse contemporaine, Paris, Ellipses, 2002, p. 13, et M. Duchein, Histoire de l'Écosse, Paris, Fayard, 1998, p. 48.

14. Christian Auer, spécialiste de l'histoire des Highlands, définit plus précisément les Clearances comme étant «le processus par lequel, entre 1760 et 1860, des dizaines de milliers de personnes furent expulsées de leurs terres dans les Hautes Terres d'Écosse et les îles des Hébrides» (C. Auer, «La Controverse de Dunrodin ou la résurgence des Highland Clearances, 1976-1977», Études écossaises, n 14, Grenoble, Ellug, 2011, p. 83.) 
environs, aida le jeune McTaggart à avoir accès à des collections particulières et lui fournit également une lettre de recommandation pour rencontrer le portraitiste Daniel Macnee (1806-1882), artiste en vogue à Glasgow. McTaggart n'étudia pas avec Macnee mais, suivant ses conseils, s'enrôla comme élève à la Trustees' Academy, centre de formation artistique ouvert à Édimbourg en 1760. Sous la direction du peintre Robert Scott Lauder (1803-1869), il étudia dans cette institution de 1852 à 1858 aux côtés de quelques-uns des plus talentueux peintres de sa génération tels Peter Graham (1836-1921), George Paul Chalmers (1833-1878) et John Pettie (1839-93) qui devint un ami intime de Mc Taggart ${ }^{15}$. Durant sa formation à la Trustees' Academy, McTaggart remporta au total quatre prix pour ses dessins et ses tableaux ${ }^{16}$. En été, lors de la période de fermeture annuelle de la Trustees' Academy, le jeune artiste quittait Édimbourg pour partir à la recherche de clients désireux de faire appel à ses talents de portraitiste. Les tableaux qu'il exécuta durant cette période connurent un franc succès : à Glasgow, pendant l'été 1852, il obtint plusieurs commandes et quatre étés consécutifs, de 1853 à 1856, il partit travailler à Dublin où il comptait une clientèle croissante. Son talent ne tarda pas non plus à être reconnu par les artistes de la Royal Scottish Academy qui l'élurent membre associé de cette institution en 1859, après qu'il eut obtenu des critiques élogieuses pour les trois tableaux envoyés aux expositions annuelles de 1858 et $1859^{17}$. McTaggart fut élu académicien de la Royal Scottish Academy en 1870 et, tout au long de sa carrière, y présenta régulièrement des œuvres à l'occasion de l'exposition annuelle. Sans pour autant totalement cesser de réaliser des portraits, il se spécialisa dans la peinture de paysages et réalisa également des scènes de genre; il avait une prédilection pour les paysages marins dans lesquels il aimait représenter des enfants au premier plan ${ }^{\mathbf{1 8}}$.

Ce ne fut qu'à la fin des années 1870 qu'il se mit à peindre des œuvres dans un style qui lui était propre. Auparavant, ses toiles présentaient des

15. La formation des jeunes artistes étudiant à la Trustees' Academy sous la direction de Robert Scott Lauder a fait l'objet d'une étude approfondie par Lindsay Errington dans Master class, Robert Scott Lauder and his pupils, Édimbourg, National Galleries of Scotland, 1983.

16. En 1855 et 1856, il gagna un second prix pour un dessin d'après une statue antique. En 1857, pour le même type de composition, il remporta le premier prix. En 1855, il reçut un troisième prix pour une peinture d'après modèle vivant et l'année suivante il décrocha le premier prix dans cette catégorie.

17. En 1858, McTaggart envoya deux tableaux intitulés The Sleeper and the Watcher (œuvre non localisée) et The Dispute (œuvre non localisée); en 1859, il exposa Going to Sea (Fife Council Museums, Kirkcaldy Museum and Art Gallery, Kirkcaldy).

18. Les enfants comptaient aussi parmi ses sujets de prédilection. Les tableaux qu'il consacra aux enfants ont fait l'objet d'une exposition en 2010 au musée de Kirkcaldy (Mc Taggart's Children: a centenary celebration, du 3 juillet 2010 au 3 octobre 2010, Kirkcaldy Museum and Art Gallery, Kirkcaldy). 
caractéristiques évoquant les tableaux des peintres préraphaélites qu'il avait pu admirer en 1857 lorsqu'il alla à Manchester à l'occasion de l'exposition intitulée «Art treasures of the United Kingdom ${ }^{19}$ ». En effet, la vivacité du coloris et la minutie avec laquelle furent peints les personnages et le paysage au premier plan des toiles nommées Spring et Dora rappellent les œuvres des préraphaélites. À la manière de ces derniers, qui prenaient souvent des œuvres littéraires comme sujet, McTaggart s'inspira d'un poème tiré du recueil English Idyls (1842) de Lord Tennyson, lorsqu'il peignit Dora. Dans son ouvrage consacré à McTaggart, Lindsay Errington a noté que, pour cette toile, le peintre fut influencé par deux œuvres de John Everett Millais (1829-1896), Autumn Leaves et The Blind Girl $^{20}$. L'influence de Millais est tout aussi apparente dans The Wreck of the Hesperus, toile intitulée d'après un poème de Henry Longfellow. Cette œuvre, qui représente un pêcheur découvrant sur le rivage le corps sans vie d'une jeune femme et les débris d'un bateau ayant fait naufrage, rappelle l'Ophelia de Millais. Durant les années 1860, certains tableaux montrent qu'il ne fut pas seulement influencé par les préraphaélites, puisque des œuvres telles The Orange Girl et Puir Weans, représentant des enfants pieds nus et vêtus de haillons, évoquent les œuvres des peintres réalistes qui prirent fréquemment pour sujet la misère des ouvriers et de leurs enfants ${ }^{21}$. Néanmoins, McTaggart s'affranchit progressivement de l'influence des préraphaélites et des peintres réalistes pour créer son propre style. Dès la fin des années 1870 , ses paysages ne sont plus peints avec le souci d'un rendu minutieux des détails; les formes, qui jusque-là se caractérisaient par des contours nets, s'estompent et sont obtenues par les touches et la juxtaposition des couleurs. Le peintre exécutait rapidement ses tableaux en peignant en plein air afin de mieux saisir les effets de lumières et de couleurs. C'est en raison de son style et de sa technique que ses tableaux datant de la fin des années 1870 sont considérés comme

19. La confrérie préraphaélite fut fondée à Londres en 1848 par trois peintres, William Holman Hunt (1827-1910), John Everett Millais et Dante Gabriel Rossetti (1828-1882), en réaction aux conventions académiques et aux productions artistiques contemporaines. Ces artistes souhaitaient revenir à une peinture semblable à celle des artistes ayant exercé avant Raphaël (1483-1520). Ils prirent pour modèle le style des primitifs italiens et, pour leurs sujets, ils s'inspirèrent souvent de la Bible, du Moyen Âge et d'ouvrages littéraires. Sur McTaggart et l'exposition «Art treasures of the United Kingdom», voir P. Kvaerne, William Mc Taggart 1835-1910. Singing Songs of the Scottish Heart, Édimbourg, Atelier Books, p. 30.

20. D'après L. Errington : «It is not too much to say that memories of these two paintings, the one a dusk scene with close-up views of unreadable childish faces, the other a long-sighted gaze past two children into a vast spread of sunlit meadow, haunted McTaggart's imagination for a whole decade.» (L. Errington, William McTaggart 1835-1910, Édimbourg, National Gallery of Scotland, p. 40.)

21. Ces toiles de McTaggart rappellent notamment Les Casseurs de Pierres (1849, œuvre détruite) et L'Aumône d'un Mendiant (1868, The Burrell Collection, Glasgow) de Gustave Courbet (1819-1877). 
s'inscrivant dans le courant impressionniste ${ }^{22}$. Déjà, en 1888, Walter Armstrong comparait les toiles de McTaggart à celles des impressionnistes puisqu'il écrivait : «of late years he [McTaggart] has outdone the impressionists in the looseness of his handling ${ }^{23} »$. Aujourd'hui encore, les spécialistes soulignent les ressemblances entre les œuvres de McTaggart et celles des impressionnistes; Kenneth McConkey estime même qu'il fait partie des artistes ayant contribué de manière significative au développement de la peinture impressionniste en Grande-Bretagne ${ }^{24}$.

La carrière de McTaggart s'étendit sur un demi-siècle, durant une période d'activité artistique intense marquant une transition dans l'histoire de l'art pictural écossais ${ }^{25}$. Lorsqu'il commença à peindre au début des années 1850, la majorité des paysagistes écossais contemporains était spécialisée dans la représentation des vues des Highlands. Horatio McCulloch, le paysagiste romantique le plus prisé d'Écosse depuis qu'il avait commencé à peindre des paysages des Hautes Terres à partir des années 1830, exerçait encore et comptait plusieurs disciples parmi les peintres de la génération de McTaggart. Entre autres, Joseph Denovan Adam (1841-1896), Peter Graham (1836-1921) et Joseph Farquharson (1846-1935) imitèrent le style et les sujets des tableaux de McCulloch : à la manière de ce dernier, ils réalisèrent essentiellement des paysages sauvages des Highlands sous un ciel d'orage, représentant des châteaux en ruine, des lacs, des montagnes aux pics acérés et généralement peuplés de quelques hommes vêtus d'un kilt, de moutons, de cerfs ou de vaches de la race Highland cattle. L'engouement des paysagistes écossais pour les Highlands fut tel qu'un critique d'art s'étant rendu aux expositions organisées par la Royal Scottish Academy et le Glasgow Institute en 1879 écrivit : «The rugged mountain tops and the fertile straths and glens offer too favourable inducements to the painter to be lightly passed over, and a great proportion of the Scotch School is absorbed in their representa-

22. Si l'on sait avec certitude que McTaggart avait pu observer des tableaux des préraphaélites et des peintres réalistes français et qu'il avait été influencé par leurs œuvres, rien ne permet d'affirmer qu'il avait été en contact avec les toiles des impressionnistes lorsqu'il adopta un nouveau style à la fin des années 1870. Voir M. Macdonald, Scottish Art, Londres, Thames and Hudson, 2000, p. 121.

23. W. Armstrong, Scottish Painters. A Critical study, Londres, 1888, p. 74. Tout au long du xx siècle, la plupart des historiens de l'art ont comparé McTaggart aux impressionnistes. Entre autres, Ritchie a affirmé : «McTaggart is the most original painter Scotland produced in the nineteenth century. [...] by 1875 [he] had found his own impressionist technique and thereafter painted the Scottish landscape and coast with penetrating understanding of local effects of light and atmosphere.» (A. Carnduff Ritchie, Masters of British painting 1800 to 1950 (1956), Arno Press, 1980, p. 88.)

24. K. McConkey, Impressionism in Britain, Londres, Barbican Art Gallery, 1995, p. 7.

25. Stanley Cursiter a affirmé que l'œuvre de McTaggart «marks a great division between the earlier and later aspects of Scottish art» (S. Cursiter, Scottish art to the close of the nineteenth century, Londres, George G. Harrap \& Co., 1949, p. 125). 
tion ${ }^{26}$.» John Morrison a rappelé qu'il était alors difficile pour les jeunes paysagistes écossais de ne pas se conformer au style de McCulloch et de ne pas peindre des vues des Highlands, car cela revenait à rejeter l'identité écossaise :

The nineteenth-century creation of a Highland identity for the whole of the country was internalised by Scots [...]. If not actually treason, resistance to Highlandism was certainly viewed in artistic circles as a conscious attack on the entire underpinning of national cultural identity. [...] Authenticity meant Highland and in this climate any resistance to the hegemony of Highlandism as Scotland's visual identity challenged not a cultural paradigm but the very identity of the country ${ }^{27}$.

Néanmoins, plusieurs artistes écossais refusaient de peindre des tableaux suivant la manière de McCulloch. À son retour en Écosse en 1869, après avoir étudié en Hollande dans l'atelier de Gerrit Alexander Mollinger (1836-1867), Sir George Reid (1841-1913) délaissa les paysages des Highlands et le style des peintres romantiques qu'il avait adopté à ses débuts pour peindre des vues des Lowlands, tels Autumn et Montrose, dans un style comparable à celui des peintres réalistes hollandais et français contemporains. Les tableaux qu'il peignit à son retour de Hollande furent critiqués avec virulence dans la presse et par les membres de la Royal Scottish Academy ${ }^{28}$. Toutefois, Reid ne renonça pas à son nouveau style; dans son essai intitule Thoughts on Art, and Notes on the Exhibition of the Royal Scottish Academy of 1868, il n'hésita pas à dénoncer le style de McCulloch et de ses disciples :

26. G. R. Halkett, «Royal Scottish Academy and Glasgow Institute exhibitions », $2^{\mathrm{e}}$ partie, The Magazine of Art, 1879, p. 266.

27. J. Morrison, Painting the Nation. Identity and nationalism in Scottish painting, 1800-1920, Édimbourg, Edinburgh University Press, 2003, p. 147-148.

28. Morrison explique : «In 1866 Reid was viewed by senior figures in the R.S.A. as a promising landscape painter in the late Romantic manner of Horatio McCulloch (1805-67). Indeed Reid had come directly to McCulloch's attention immediately before setting off for Europe when the older artist, unbidden, had "corrected" the painting of the sky in one of Reid's landscapes as it hung on the walls of the R.S.A. annual exhibition. There was mild disquiet among R.S.A. members at Reid's decision to leave Scotland and study with Mollinger. [...] When Reid returned from Holland, having abandoned his images of castles, storms and mountains, and embraced Mollinger's Realist subject matter of rural genre, field labour and agricultural landscape, along with the Dutchman's penchant for heavy dragged paint and overtly emotional handling, there was genuine anger. Reid was vilified in the press, then summoned by Sir George Harvey (1806-76), the President of the R.S.A., and castigated for "looking at nature through Mollinger's eyes". » (J. Morrison, «Holland and France: prototype and paradigm for nineteenth-century Scottish Art» [en ligne], Fournal of Irish Scottish Studies, vol. 2, $\mathrm{n}^{\circ}$ 1, 2008, p. 123. Disponible sur <www.abdn.ac.uk/riiss/JISS/2.1/2.1_Morrison. pdf> [consulté le 23/06/2012].) 
Macculloch had no real feeling for the majesty and mystery of the noble scenery he chose as the subject of his art. Hardness and conventionality were the characteristics of all his later works. His method of studying nature was too superficial to enable him to reach to any high degree of excellence $[\ldots]$. His influence on the Scottish School of landscape has been most hurtful, as may be seen in the efforts of a plentiful crop of imitators, who, instead of looking at nature through their own eyes, look at it through the medium of Macculloch's works ${ }^{29}$.

Dans cet ouvrage, Reid invitait les jeunes artistes écossais à suivre son exemple et à s'inspirer des peintres réalistes français et hollandais pour peindre des paysages écossais ${ }^{30}$. Les artistes connus sous le nom de Glasgow Boys ${ }^{31}$ partagèrent avec Reid la volonté de transformer la peinture écossaise. À l'instar de ce dernier, ces jeunes peintres critiquaient ouvertement les académiciens, qu'ils surnommaient les «gluepots» en raison de leur utilisation excessive d'un vernis à base de bitume qui servait à donner à leurs tableaux un aspect de patine naturelle. Ils leur reprochaient aussi de ne pas peindre en plein air et de ne pas représenter fidèlement les paysages et les habitants de l'Écosse contemporaine ${ }^{32}$. À la manière des peintres naturalistes qu'ils avaient pu côtoyer lors de leur formation dans les ateliers parisiens et à l'occasion de leurs séjours dans la communauté d'artistes établie à Grez-Sur-Loing, les Glasgow Boys puisèrent leurs motifs dans les communautés villageoises. Tout au long des années 1880, ils peignirent des scènes de genre et des paysages repré-

29. Veri Vindex, Thoughts on art, and notes on the exhibition of the Royal Scottish Academy of 1868, Édimbourg, Alexander Hislop and Company, 1868, p. 40. Reid critiqua encore plus sévèrement les tableaux d'Arthur Perigal (1816-1884) : «We see in the case of Perigal a confirmation of what we have said of Macculloch, that he was conventional and mannered in spite of his great cleverness and dexterity. The disease appears fearfully intensified in the imitator. It has run its course, as might have been expected; and in Perigal we see the dregs of Maccullochism.» (Ibid., p. 43-44)

30. Ibid., p. 52.

31. Les spécialistes sont en désaccord sur le nombre exact de peintres faisant partie des Glasgow Boys. Dans son ouvrage The Glasgow Boys. The Glasgow school of painting 1875-1895, Roger Billcliffe compte quatorze peintres : Joseph Crawhall (1861-1913), Thomas Millie Dow (1848-1919), James Guthrie (1859-1930), George Henry (1858-1943), Edward Atkinson Hornel (1864-1933), William Kennedy (1859-1918), Sir John Lavery (1856-1941), William York MacGregor (1855-1923), Alexander Mann (1853-1908), Arthur Melville (1855-1904), James Paterson (1854-1932), Alexander Roche (1861-1921), Robert Macaulay Stevenson (1854-1952) et Edward Arthur Walton (1860-1922). Quant à eux, les auteurs du catalogue de l'exposition Pioneering painters. The Glasgow Boys 1880-1900 répertorient au total dix-huit peintres. Outre les peintres énoncés précédemment, ils considèrent que David Gauld (1865-1936), James Whitelaw Hamilton (1860-1930), James Nairn (1859-1904) et James Stuart Park (1862-1933) étaient également des membres des Glasgow Boys.

32. Selon Billcliffe, les peintres de Glasgow estimaient que les paysages des peintres écossais romantiques «were all theatrical backcloths, survivals from an earlier age, painted without capturing the true value of the landscape, which could as much have been Switzerland as Scotland» (R. Billcliffe, The Glasgow Boys. The Glasgow school of painting 1875-1895, Londres, John Murray, 2002, p. 27). 
sentant les comtés agricoles des Lowlands dans un style évoquant celui du peintre Jules Bastien-Lepage (1848-1884) qu'ils admirèrent profondément à leurs débuts ${ }^{33}$. Avec leurs tableaux, les Glasgow Boys souhaitaient rappeler que l'Écosse ne se compose pas seulement des Highlands, mais comprend aussi les Lowlands. William McTaggart refusa lui aussi de peindre des tableaux conformes aux traditions des paysagistes romantiques écossais et affirma :

It is not grand scenery that makes a fine landscape. You don't find the best artists working in the Alps. It's the heart that's the thing. You want to express something that appeals to common humanity, not something extraordinary... Of course you can understand the older Scottish landscape painters going to the Highlands in the footsteps of Sir Walter Scott, but humanity's the thing! ${ }^{34}$

Bien que cette remarque évoque les critiques que Reid et les Glasgow Boys adressèrent à l'encontre des paysagistes romantiques et qu'il souhaitât, lui aussi, représenter les paysages de l'Écosse sans fard, McTaggart n'en réalisa pas moins des tableaux au style et aux sujets très différents. Reid et les Glasgow Boys, durant les années 1880, exécutèrent essentiellement des vues des Lowlands dont les moindres détails sont peints avec minutie, tandis que McTaggart préférait peindre des paysages des Highlands se caractérisant par un coloris vif et une peinture apposée rapidement en couche épaisse où il est, parfois, à peine possible de distinguer les personnages du paysage. Il ne cessa cependant jamais de représenter des vues des comtés du sud de l'Écosse puisqu'il alla régulièrement peindre dans le village de Carnoustie, sur la côte est au nord de Dundee, et qu'il s'inspira du jardin de sa propriété de Broomieknowe, située à quelques kilomètres d'Édimbourg. Ce furent toutefois les paysages côtiers du nordouest de l'Écosse qui l'inspirèrent le plus; jusqu'à sa mort, en 1910, il eut pour habitude de retourner peindre dans les Highlands presque chaque été. Il alla essentiellement à Campbeltown, Carradale, Machrihanish et Glenramskill, ports de la péninsule de Kintyre; il travailla également à Tarbet, sur les îles d'Aran et l'île d'Iona. À la différence des Glasgow Boys

\footnotetext{
33. Avec leur série de tableaux consacrés aux Lowlands, les peintres de l'école de Glasgow ont connu un immense succès auprès des collectionneurs et des critiques en Grande-Bretagne et sur le continent européen. Même les membres de la Royal Scottish Academy reconnurent leur talent et en 1888 Guthrie fut le premier Glasgow Boy à être élu membre associé de cette institution. Par la suite, les principaux représentants de l'école de Glasgow devinrent académiciens et, en 1902, Guthrie en fut même élu Président. À partir des années 1890, les Glasgow Boys adoptèrent des styles différents pour peindre des tableaux ayant des thèmes divers : ainsi, Henry et Hornel consacrèrent des toiles à des sujets inspirés de leur voyage au Japon; pour sa part, Lavery se spécialisa dans l'art du portrait, tandis que Melville et Crawhall, tous deux adeptes de l'aquarelle, peignirent respectivement un grand nombre de paysages d'Afrique du Nord et de sujets animaliers.

34. Cité dans The Discovery of Scotland, ouvr. cité, p. 137.
} 
et de Reid, McTaggart n'insistait pas sur l'idée que l'Écosse se compose de deux régions bien distinctes. Ses tableaux se démarquent aussi de ceux des disciples de McGulloch, car ils représentent presque exclusivement des marines et non des paysages des montagnes des Hautes Terres.

Dans les toiles que McTaggart a consacrées à la côte ouest de l'Écosse, paysage et histoire sont souvent associés ${ }^{35}$. C'est essentiellement avec le titre du tableau que le peintre établit un lien entre l'histoire des Highlands et le lieu représenté. Ainsi The Emigrants Leaving the Hebrides, The Emigrants - America, et The Sailing of the Emigrant Ship font référence à l'exode massif des habitants des Highlands durant le XVIII ${ }^{\mathrm{e}}$ siècle et la première moitié du XIX". Les toiles intitulées The Coming of Saint Columba et The Preaching of Saint Columba rappellent quant à elles que les régions côtières de l'ouest et du nord jouèrent un rôle essentiel dans l'histoire de l'Écosse puisqu'au $\mathrm{VI}^{\mathrm{e}}$ siècle, ce fut à partir de l'île d'Iona que se propagea le christianisme à travers les différents royaumes établis sur le territoire écossais. Membre de la famille royale d'Irlande, Colomba quitta son pays natal pour tenter d'évangéliser les peuples scots et pictes. À son arrivée, ce fut sur l'île d'Iona qu'il décida de fonder la première abbaye chrétienne en Écosse, et ainsi que l'a affirmé Michel Duchein : «Le rayonnement du nouvel établissement fut immédiat et lointain ${ }^{36}$.» Après son décès vers 597, les moines de l'abbaye d'Iona continuèrent son œuvre et fondèrent plusieurs autres abbayes, notamment à Melrose et à Inchmahome, afin de convertir les Pictes au christianisme. Saint Colomba compte désormais parmi les personnages les plus marquants de l'histoire de l'Écosse, d'après Nigel Trenter : «He was, by any reckoning, one of the truly great, and a person who had the most major influence on Scotland's story, to rank with, if not above, MacBeth, Margaret, David the first, Wallace, Bruce, James the Fourth, John Knox and the others ${ }^{37}$.»

Au total, McTaggart consacra trois œuvres à l'arrivée du saint en Écosse : dans sa première toile, il a représenté le missionnaire alors qu’il est sur le point d'atteindre la côte écossaise; un second paysage désigne l'endroit où il a débarqué; enfin, la troisième toile est dédiée au premier sermon du saint en Écosse. Les spécialistes pensent que son attachement profond à la culture gaélique dont il était issu et ses visites fréquentes sur la côte ouest l'incitèrent à célébrer, dans ses paysages, cet événement

35. Ainsi que l'a noté Kvaerne, durant les années 1860, des vues des Highlands par McTaggart peuvent aussi être associées à des œuvres littéraires d'Allan Ramsay (1686-1758) et de Robert Burns (1759-1796) (William Mc Taggart 1835-1910. Singing Songs of the Scottish Heart, ouvr. cité, p. 58).

36. Histoire de l'Écosse, ouvr. cité, p. 48.

37. N. Tranter, The Story of Scotland, Glasgow, Neil Wilson Publishing, 2003, p. 10. 
majeur de l'histoire de l'Écosse ${ }^{38}$. Il semble aussi que l'intérêt des artistes écossais contemporains pour l'histoire et les légendes gaéliques ${ }^{39}$, ainsi que la publication d'une nouvelle édition de la vie de saint Colomba par Adamnan aient inspiré la réalisation de la série d'œuvres commémorant la venue du saint dans les Highlands. En effet, ce fut en 1895 que McTaggart réalisa The Coming of Saint Columba et The Preaching of Saint Columba, date à laquelle parut Prophecies Miracles and Visions of St Columba, ouvrage dont le peintre fit l'acquisition ${ }^{40}$. La troisième et dernière œuvre qu'il dédia au missionnaire fut exécutée en 1904 : il s'agit de l'aquarelle When Saint Columba Landed. Le personnage de Colomba n'apparaît que dans un seul de ces paysages : dans son tableau The Preaching of Saint Columba, McTaggart a représenté le saint portant une tenue blanche en compagnie d'autres personnages vêtus eux aussi de blanc, certainement quelques-uns des douze moines qui l'accompagnèrent lors de son périple en Écosse. Colomba vient tout juste d'arriver en Écosse comme en attestent les trois bateaux amarrés sur la plage. Pour prêcher, le missionnaire se tient au bord de la mer, les bras levés et tendus; un nombre important de personnages sont témoins de son arrivée et assistent à son sermon, tandis qu'au premier plan, sur un promontoire, des enfants sont occupés à jouer et ne prêtent pas attention à la scène qui se déroule autour d'eux. Dans The Coming of Saint Columba, la présence de Colomba est suggérée par les trois bateaux figurant à l'arrière plan; un couple et un enfant sont allongés sur l'herbe d'un promontoire surplombant la plage, l'homme observe l'arrivée imminente des navires. Dans l'aquarelle When Saint Columba Landed, rien ne signale la présence du missionnaire; aucun personnage n'est représenté dans cette œuvre où le peintre a reproduit une partie du paysage figurant en bas dans l'angle droit du tableau The Coming of Saint Columba. Seul le titre de l'aquarelle laisse penser que l'artiste a souhaité peindre l'endroit où le saint est censé être arrivé. Comme l'ont souligné plusieurs spécialistes, McTaggart n'a pas représenté un paysage de l'île d'Iona même si, en Écosse, c'est le lieu le plus étroitement associé à saint Colomba; pour ses trois peintures, il a préféré peindre une

38. Voir notamment William McTaggart 1835-1910. Singing Songs of the Scottish Heart, ouvr. cité, p. 203.

39. Au cours des années 1870, Robert Gibb (1845-1932) peignit deux tableaux représentant saint Colomba. Le premier s'intitule Columba in Sight of Iona (œuvre non localisée) et date de 1874; le second, Death of St Columba (œuvre non localisée) fut exécuté en 1876. De plus, comme l'a rappelé Murdo Macdonald, au moment où McTaggart réalisait sa série de tableaux sur le missionnaire, Patrick Geddes (1854-1932) proposa d'ériger à Édimbourg une statue en l'honneur du saint (Scottish Art, ouvr. cité, p. 125).

40. P. Kvaerne a précisé que «among McTaggart's books was a new translation [...], of Saint Adamnan's Prophecies Miracles and Visions of St Columba as well as other books on Celtic culture» (William Mc Taggart 1835-1910. Singing Songs of the Scottish Heart, ouvr. cité, p. 203). 
vue de la baie de Gauldrons, sur la péninsule de Kintyre, à proximité de l'endroit où le missionnaire aurait débarqué pour la première fois en Écosse $^{41}$. Avec cette série de peintures, McTaggart célèbre un épisode glorieux de l'histoire des Highlands, région choisie pour être le centre de la civilisation chrétienne en Écosse. Ces œuvres s'inscrivent dans le mouvement culturel du renouveau celtique qui apparut dans la peinture écossaise à la fin du XIX ${ }^{\mathrm{e}}$ siècle, mais se démarquent des tableaux que les peintres écossais de cette période consacrèrent à la culture et civilisation gaélique ancienne, car elles privilégient les faits historiques aux légendes. En effet, John Duncan (1866-1945) et Alice Boyd (1825-1897), qui ont tous deux exécuté des tableaux avec saint Colomba comme sujet, se sont inspirés d'une des nombreuses légendes relatées par Adamnan ${ }^{42}$ lorsqu'ils ont peint leurs toiles respectivement intitulées Saint Columba Bidding Farewell to the White Horse et St Columba's Farewell to the White Horse. De plus, contrairement à des tableaux tels The Druids: Bringing Home the Mistletoe de Henry (1858-1943) et de Hornel (1864-1933) et The Awakening of Cuchullin de John Duncan, dans lesquels les tenues et les bijoux, inspirés de ceux portés au début de la civilisation celte ${ }^{43}$, permettent aux spectateurs de situer dans le temps les scènes représentées, les peintures de McTaggart sont intemporelles : à l'exception du titre, aucun élément de la composition ne permet de définir un contexte temporel précis. Dans les deux tableaux sur lesquels figurent des personnages, ceux-ci sont à peine visibles et le peintre n'a pas essayé d'imaginer ou de recréer les tenues que pouvaient porter les premiers missionnaires chrétiens au $\mathrm{VI}^{\mathrm{e}}$ siècle. Ainsi que l'a affirmé l'historien de l'art Murdo Macdonald :

41. Errington, Morrison et Kvaerne ont identifié le paysage comme étant la baie de Gauldrons, au sud-ouest de Macrahanish (William McTaggart 1835-1910, ouvr. cité, p. 110 ; Painting the Nation, ouvr. cité, p. 208; William Mc Taggart 1835-1910. Singing Songs of the Scottish Heart, ouvr. cité, p. 199). Saint Colomba n'a pas débarqué à la baie de Gauldrons, mais plus précisément à Southend qui se trouve sur la péninsule de Kintyre. Nigel Tranter explique que saint Colomba ne souhaita pas s'établir sur la péninsule de Kintyre, car il pouvait encore apercevoir les côtes de l'Irlande. Il décida de continuer à naviguer plus au nord, le long de la côte ouest de l'Écosse, et s'installa sur l'île d'Iona (The Story of Scotland, ouvr. cité, p. 8-9).

42. Il s'agit de la légende du cheval blanc de saint Colomba, selon laquelle l'animal, pressentant le décès de son maître, se mit à pleurer.

43. John Morrison a constaté que plusieurs peintres dont les œuvres s'inscrivent dans le mouvement du renouveau celtique se sont inspirés d'ouvrages reproduisant des objets et bijoux du début de la civilisation celtique. À propos de The Druids: Bringing Home the Mistletoe, il a noté : «[Henry and Hornel] were clearly concerned to make the picture historically accurate. [...] In company with Henry, Hornel undertook numerous searches for Celtic remains in the Galloway countryside before starting work on The Druids. Their specific knowledge of Scottish "Celtic" art is clearly demonstrated throughout the painting. Much of the symbolism is drawn from Pictish carved stones.» (Painting the Nation, ouvr. cité, p. 194.) 
McTaggart [...] integrated his Highland background into his work, making no distinction between his modern self as an artist and the traditions of his folk. [...] It [the series of paintings devoted to Saint Columba] is a timeless image of a Celtic space which is both ancient and modern ${ }^{44}$.

En revanche, dans la série de tableaux qu'il a consacrée à l'émigration des habitants des Highlands, certains éléments de la composition permettent de situer les scènes représentées durant la première moitié du XIX ${ }^{\mathrm{e}}$ siècle.

La première œuvre de McTaggart évoquant l'exode des Highlanders date de 1864 et s'intitule Word from the West. Au cours des années 1880 et 1890, le peintre a abordé ce thème dans pas moins de six tableaux : Away o'er the Sea-Hope's Whisper (1889), The Emigrants - America (1891-1894), A Sprig of Heather - Farewell to the Emigrants (1893), The Sailing of the Emigrant Ship (1895), ainsi que dans deux toiles intitulées Emigrants Leaving the Hebrides (1883-1889 et 1891-1898). Dans ces tableaux, McTaggart fait allusion à la vague d'émigration qui eut lieu dans les Highlands au milieu du XIX ${ }^{\mathrm{e}}$ siècle : le peintre a représenté un navire à voiles, semblable à ceux empruntés par les émigrés lors de leur traversée de l'Atlantique durant la première moitié du XIX ${ }^{\mathrm{e}}$ siècle, mais qui avaient été depuis remplacés par des bateaux à vapeur ${ }^{45}$. De plus, lorsque McTaggart exécuta ces toiles, les navires à destination des États-Unis et du Canada ne faisaient plus escale le long de la côte ouest pour embarquer des voyageurs, mais partaient notamment de Glasgow. L'artiste a confié à son gendre et biographe James Caw que, durant son enfance, il avait été témoin de scènes semblables à celles figurant dans cette série de tableaux ${ }^{46}$. Issu de la classe paysanne des crofters qui émigra massivement durant le XIX ${ }^{\mathrm{e}}$ siècle, il fut personnellement concerné par le départ de membres de sa famille puisque sa sœur Barbara et son mari partirent vivre en Amérique du Nord.

Au cours des XVIII ${ }^{\mathrm{e}}$ et XIX ${ }^{\mathrm{e}}$ siècles, il y eut trois vagues successives d'émigration des habitants des Highlands vers les États-Unis, le Canada et l'Australie $^{47}$ : la première vague eut lieu entre les années 1760 et le début

44. Scottish Art, ouvr. cité, p. 122. Cette remarque fait écho à celle de Kvaerne, qui a écrit au sujet des paysages célébrant la venue de saint Colomba : «They are down-to-earth illustrations of real events, inserted into real landscapes - and yet a landscape which is timeless and intensely poetic.» (William Mc Taggart 1835-1910. Singing Songs of the Scottish Heart, ouvr. cité, p. 203.)

45. Ibid., p. 197.

46. Sir J. Caw explique : «In his youth he had seen many of his friends and acquaintances leave for America to find there the scope and the land to live on, which they had been denied at home. He had seen more than once that suggestively named ship "The Gleaner" sail from Campbeltown Loch crowded with emigrants, cleared or clearing from Argyll.» (J. Caw, William Mc Taggart, Glasgow, James Maclehose and Sons, 1917, p. 169.)

47. Sur l'émigration des habitants des Highlands en Australie, voir C. Auer, " "The Lazy Highlander and the industrious colonist" : la perception ambivalente du Highlander dans les années 1850 ", 
des guerres napoléoniennes; l'exode reprit après 1815 et dura jusqu'à 1840 ; il y eut encore un nombre important d'émigrés au milieu du XIX ${ }^{\mathrm{e}}$ siècle, lorsque les Hautes Terres souffrirent à nouveau de la famine à partir de 1846 suite à de mauvaises récoltes de la pomme terre, atteinte de la maladie de la brunissure ${ }^{48}$. Les causes de l'exode des Highlanders aux XVIII ${ }^{\mathrm{e}}$ et XIX ${ }^{\mathrm{e}}$ siècles sont multiples, selon Thomas Devine : «Clearances, commercialisation, demographic pressures, famine, economic collapse and landlordism all had an impact on the emigration process ${ }^{49}$ ». L'échec de la rébellion jacobite de 1745 ne fit qu'accélérer le processus de désintégration des clans, déjà bien avancé. Dès lors, les chefs de clans s'absentèrent plus fréquemment de leurs propriétés; ils se rapprochèrent de la noblesse des Lowlands et aspirèrent au confort dont jouissaient ces derniers. Afin de financer leur nouveau mode de vie, ils louèrent leurs terres à des prix plus élevés; il s'ensuivit une inflation galopante des loyers qui eut des répercussions immédiates et profondes dans les Highlands, causant une première vague d'émigration. Plutôt que de continuer à fractionner leur propriété en petites parcelles de terre comme ils l'avaient fait traditionnellement, les chefs de clans en optimisèrent la rentabilité en y développant l'élevage intensif de moutons. Avec l'essor des industries textiles dans les Lowlands et en Angleterre, la demande de laine augmenta considérablement et les élevages ovins s'avérèrent plus lucratifs que toute autre activité. Les premiers élevages de moutons de races Cheviot et Linton furent introduits au début des années 1760 dans les comtés de Perth et d'Argyll au sud des Highlands; avant la fin du siècle, ils gagnèrent l'ensemble de la région ${ }^{50}$. L'élevage intensif engendra le déplacement de la population, cette activité nécessitant de grands espaces et peu de main-d'œuvre ${ }^{51}$. De plus, à partir des années 1840, les propriétaires terriens commencèrent à créer un nombre croissant de très grandes réserves de chasse aux cerfs qui occasionnèrent de nouvelles expropria-

dans M. Munro-Landi (éd.), L'Écosse et ses doubles ancien monde-nouveau monde; Old world-new world Scotland and its doubles, Paris, L'Harmattan, 2010, p. 165-176.

48. Les habitants des Hautes Terres souffrirent de famine en 1836 et 1837, années de mauvaises récoltes.

49. T. M. Devine, Clanship to Crofters' War. The transformation of the Scottish Highlands, p. 177. Sur les causes de l'émigration, voir aussi les articles de M. I. Adam, «The Highland emigration of $1770 »$, The Scottish Historical Review, n 16, 1919, p. 280-293 et «The Causes of Highland emigrations of 1783-1803», The Scottish Historical Review, n 17, 1920, p. 73-89.

50. Devine estime qu'entre 1811 et 1854, dans le comté de Ross, le nombre de moutons fut multiplié par cinq; dans le comté d'Inverness, il passa de 154000 à 542000 (Clanship to Crofters' War, ouvr. cité, p. 75-76).

51. L'historien John Prebble a précisé que «four shepherds, their dogs and three thousand sheep [...] occupied land that had once supported five townships» (J. Prebble, The Highland Clearances, ouvr. cité, p. 28). 
tions ${ }^{52}$. Parfois, en guise de compensation, les propriétaires proposèrent aux membres de leurs clans des petites parcelles de terre à la périphérie de leurs propriétés; ce fut notamment le cas le long de la côte ouest et sur les îles Hébrides. Toutefois, le plus souvent, les fermiers furent expulsés de leurs terres et contraints à émigrer. Les personnes chargées par les chefs de clan d'organiser les expropriations n'hésitèrent pas à avoir recours à la violence, comme en témoigne le récit de la jeune Betsy Mackay :

Our family was very reluctant to leave $[\ldots]$ and stayed for some time, but the burning party came round and set fire to our house at both ends, reducing to ashes whatever remained within the walls. The people had to escape for their lives, some of them losing all their clothes except what they had on their backs. The people were told they could go where they liked, provided they did not encumber the land that was by rights their own. The people were driven away like dogs who deserved no better ${ }^{53}$.

Le nombre exact d'émigrés n'a pas pu être évalué précisément, mais Devine pense qu'entre 1846 et 1857, au moins 16500 personnes partirent pour le Canada et l'Australie. Ce furent les îles de Skye, de Mull et l'ensemble des îles Hébrides qui connurent le taux d'émigration le plus élevé : la population de certaines paroisses diminua d'un tiers, voire de la moitié ${ }^{54}$.

Pour les deux versions de Emigrants Leaving the Hebrides, The Emigrants America et The Sailing of the Emigrant Ship, McTaggart a peint le même paysage, utilisant une composition presque identique à laquelle il a apporté de légères modifications et dont il a fait varier le coloris. Pour ces tableaux, le peintre a représenté des personnages — essentiellement des femmes et des enfants - venus en accompagner d'autres qui montent à bord de quatre petites embarcations qui vont les emmener jusqu'au navire, au large, et, de là, vers les États-Unis comme l'indique l'inscription «AMERICA» figurant sur une malle en bas à droite de la deuxième version de Emigrants Leaving the Hebrides datant des années 1890. Dans cette toile, de même que dans The Emigrants - America, une jeune femme agite un mouchoir blanc pour adresser un ultime salut aux émigrés, tandis que ce même personnage était assis et semblait observer passivement la scène

52. Smout a rappelé que le nombre de réserves de chasse aux cerfs augmenta tout au long du XIX ${ }^{e}$ siècle : «The number of Scottish deer forests in 1811 reckoned at only six where the deer were actively preserved had reached eighteen by 1825 and forty by 1842 [... By 1895 there were 137 forests covering more than three million acres of Highland Scotland. (T. C. Smout, «Landseer's Highlands", The Monarch of the Glen. Landseer in the Highlands, Édimbourg, National Galleries of Scotland, 2005, p. 15-16.)

53. Cité dans The Highland Clearances, ouvr. cité, p. 79.

54. Clanship to Crofters' War, ouvr. cité, p. 186. 
dans la première version du tableau. Dans A Sprig of Heather - Farewell to the Emigrants, le peintre a de nouveau représenté une jeune femme de dos agitant un mouchoir blanc en direction des émigrés qui sont à bord d'une barque qui se dirige vers le navire qui figure à l'arrière-plan. Au premier plan de Away o'er the Sea - Hope's Whisper, une femme est allongée dans l'herbe avec deux enfants; l'un d'eux semble lui chuchoter à l'oreille, tandis qu'à l'arrière-plan un navire s'éloigne de la côte. Bien que ces six œuvres semblent ne représenter le départ que d'un seul et même navire, le peintre ne fait pas référence à un événement précis; prises ensemble, ces peintures commémorent l'exode de milliers d'habitants des Highlands partis vivre en Amérique du Nord ou en Australie, loin de la terre de leurs ancêtres. Ainsi que l'écrit Lindsay Errington à propos de cette série de toiles: «[the] composition was never intended as a news style record of any one emigrant group's departure [...] [but rather] an emotionally charged reflection or recollection of Scottish Highland emigration as a whole ${ }^{55}$.» Cette impression est renforcée par les titres de chacune des œuvres ne mentionnant pas d'informations précises concernant la date, la localité représentée ou le nom du navire transportant les émigrés, ainsi que par la manière dont l'artiste a peint les personnages qui n'ont pas d'identité individuelle. En effet, dans A Sprig of Heather - Farewell to the Emigrants, la jeune femme qui occupe le premier plan de la composition apparait de dos, tandis que dans les autres tableaux l'expression du visage des personnages n'est pas visible. À l'exception de A Sprig of HeatherFarewell to the Emigrants, les personnages se fondent dans le paysage et cela laisse penser que le peintre a puisé dans ses souvenirs pour évoquer un fait passé; la représentation des personnages suggère aussi l'attachement profond des habitants des Highlands à la terre natale; le navire s'apprêtant à disparaître à l'horizon et les personnages à peine visibles semblent indiquer que, bientôt, la présence des Highlanders ne sera plus qu'un souvenir.

Cette série de tableaux évoquant des faits historiques reflète également le contexte politique contemporain. McTaggart commença à exécuter ces toiles à partir de 1883, ce qui coïncide avec une période d'agitation dans les Highlands. En 1882, des paysans établis sur la propriété de Lord MacDonald, sur l'île de Skye, refusèrent de payer leur loyer et s'opposèrent violemment aux forces de l'ordre chargées de les expulser. Ce conflit, désormais connu sous le nom de la «guerre des crofters», incita d'autres paysans des îles de Skye, Lewis, South Uist, Harris et de Tiree à entreprendre des actions similaires. En 1883, ces événements amenèrent le gouvernement britannique à constituer une commission royale présidée

55. Cité dans Scottish Art, ouvr. cité, p. 123. 
par Lord Napier, chargée d'étudier les conditions de vie de la classe paysanne dans les Highlands; après la publication du rapport de la commission, le Crofters Holdings Act fut voté en 1886 et marqua la fin des Clearances puisque la loi garantissait plus de sécurité aux paysans qui, dès lors, ne pouvaient plus être expulsés s'ils payaient leur loyer ${ }^{56}$.

McTaggart ne fut pas le seul artiste exerçant durant la seconde moitié du XIX ${ }^{\mathrm{e}}$ siècle à peindre des tableaux évoquant l'émigration des habitants des Highlands, puisque ce fut aussi le cas de John Watson Nicol (18561926) et de Thomas Faed (1826-1900 $)^{57}$. Faed a notamment exécuté trois scènes de genre — The Last of the Clan, Sunday in the Backwoods et Oh Why Have I Left My Hame? — sur ce thème. La première œuvre représente un groupe de personnages rassemblés en bord de mer assistant au départ d'émigrés; seuls ceux restant dans les Highlands sont visibles et l'attention du spectateur est plus particulièrement attirée sur un vieillard qui se trouve au centre de la composition. Sa posture — tête baissée et dos courbé - suggère l'accablement du vieil homme qui, en raison de son âge, ne peut suivre sa famille et doit rester en Écosse. Dans Sunday in the Backwoods, Faed a peint des émigrés à l'extérieur de leur nouvelle demeure en Amérique du Nord. Le visage des personnages traduit l'inquiétude et la tristesse, et dans cette toile, même les enfants semblent soucieux; l'un des personnages, une jeune femme, est malade et semble très affaiblie. Dans le troisième tableau de Faed figure un vieil homme vêtu d'un kilt, assis sur un rocher au bord d'un lac; songeur, il contemple le paysage, qui évoque celui des Hautes Terres. Pour sa part, John Watson Nicol a peint Lochaber No More, œuvre dont le titre est inspiré d'un poème d'Allan Ramsay, représentant un couple d'émigrés et leur chien à bord d'un navire. Adoptant une pose dénotant l'inquiétude et la réflexion, l'homme regarde au large, tandis que la femme s'est effondrée sur les caisses qui contiennent

56. Devine résume le contenu de la loi et son impact en ces termes : «Security of tenure for crofters was guaranteed as long as rent was paid and fair rents would be fixed by a land court; compensation for improvements was allowed to a crofter who gave up his croft or was removed from it; crofts could not be sold but might be bequeathed to a relative and, with certain restrictions, the compulsory enlargement of holdings could be considered by the land court. [...] The Crofters' Act made clearances in the old style impossible, breached the sacred rights of private property, controlled landlord-crofter relations through a government body and afforded the crofting population secure possession of their holdings. The balance of power between landlords and small tenants had been irrevocably altered by 1886.» (Clanship to Crofters' War, ouvr. cité, p. 221.)

57. Robert Herdman (1829-1888) a peint Landless and Homeless: Farewell to the Glen (1887, œuvre non localisée). Un peintre écossais, Erskine Nicol (1825-1904), a réalisé un tableau intitulé The Emigrants (1864, Tate Britain, Londres) sur le thème de l'émigration, mais plutôt que de peindre des émigrés originaires des Highlands il a représenté des Irlandais. Les peintres anglais se sont eux aussi inspirés de ce thème : entre autres, Richard Redgrave (1804-1888) et Ford Madox Brown ont peint The Emigrant's Last Sight of Home (1858, Tate Britain, Londres) et The Last of England (1852, Birmingham Museums and Art Gallery, Birmingham). 
les quelques biens qu'ils emportent, et son attitude suggère son désespoir de devoir quitter sa terre natale. Dans chacun de ces tableaux, l'expression du visage des personnages et leur pose traduisent la tristesse, la souffrance et la détresse des émigrés, ainsi que celles des membres de leur famille qui ne peuvent pas les accompagner. Les tableaux de Nicol et de Faed faisaient écho à la manière dont les écrits contemporains décrivaient le drame humain occasionné par les évictions dans les Hautes Terres. Le nombre d'articles de presse et d'ouvrages consacrés aux Clearances augmenta fortement durant la seconde moitié du XIX ${ }^{\mathrm{e}}$ siècle. La presse insista sur la brutalité des évictions et la souffrance de la population; dans les Lowlands comme en Angleterre, l'opinion publique fut émue et offrit son soutien à la cause des paysans des Highlands ${ }^{58}$. Avec sa série de tableaux évoquant l'exode des habitants des Hautes Terres, McTaggart propose une autre vision d'une période marquante dans l'histoire de la région; à la différence de la plupart des peintres et journalistes contemporains qui se focalisaient sur la notion de tragédie, il n'a pas souhaité évoquer les sentiments éprouvés par les personnes contraintes à s'exiler. Alors que ces toiles sur l'arrivée de saint Colomba dans les Highlands retracent le début de la civilisation chrétienne celtique en Écosse, ces six peintures sur le thème de l'émigration commémorent la fin de la société traditionnelle dans les Highlands. Ainsi que l'a écrit John Morrison :

These $[. .$.$] paintings may be considered together as, broadly speaking, dealing$ with the inception and demise of 1,300 years of Celtic culture in Scotland. To this extent they represent not a "Celtic revival" but rather a celebration and lament for the Gael in Scotland ${ }^{59}$.

Toutefois, même si les tableaux de McTaggart célèbrent la fin d'une ère dans l'histoire des Highlands, ces toiles dénotent un certain optimisme. Le titre Away o'er the Sea - Hope's Whisper et l'arc-en-ciel qui est visible à l'arrière plan de The Sailing of the Emigrant Ship, The Emigrants - America et de The Emigrants Leaving the Hebrides (1891-1898) traduisent l'espoir pour les émigrés de connaître des jours meilleurs une fois arrivés sur leur nouvelle terre d'accueil ${ }^{60}$.

58. Devine a affirmé : «At the time of the Sutherland clearances, southern newspapers, such as the Military Register and the Star, publicised and bitterly criticised the evictions, and similarly in the 1840s, the Free Church journal, The Witness, and the radical North British Daily Mail, were hostile towards landlordism. In the following decade there was also evidence of growing sympathy for the crofters. » (Ibid., p. 214)

59. Painting the Nation, ouvr. cité, p. 207.

60. Robin Nicholson pense qu'avec cette série de tableaux : «McTaggart is not simply suggesting that emigration offers hope, but is exploring more profound themes about the expectation of departure, the contrast between the landlocked present and a future of possibility.» (R. Nicholson, «Lochaber No More - Landscape, emigration and the Scottish artist 1849-1895» [en ligne], Nineteenth 
Notre étude des tableaux que McTaggart a consacrés à l'émigration et à la venue de saint Colomba dans les Highlands nous a permis de constater que le peintre a reconstitué à travers ses toiles quelques événements majeurs dans l'histoire de l'Écosse. Inspirées à la fois de l'histoire des Hautes Terres et du passé personnel de l'artiste, ces œuvres offrent une reconstitution originale et unique; elles proposent une alternative à la manière dont les peintres écossais contemporains et la presse représentaient les Highlands et le passé de la région. Avec ces tableaux, McTaggart rend hommage à sa terre natale qu'il quitta pour rejoindre Édimbourg en raison de sa carrière de peintre, mais à laquelle il resta profondément attaché, à l'image des personnages peuplant ses paysages.

L'œuvre de McTaggart a été redécouverte durant les années 1980 à l'occasion de la rétrospective que lui a consacrée la National Gallery of Scotland et, depuis, il compte parmi les peintres écossais les plus prisés en Écosse, alors que la plupart des paysagistes de la seconde moitié du $\mathrm{XIX}^{\mathrm{e}}$ siècle font l'objet de critiques virulentes de la part des historiens de l'art écossais ${ }^{61}$. L'absence de sentimentalisme et de clichés victoriens dans ses évocations des paysages et de l'histoire des Highlands explique le succès de ses tableaux auprès des spécialistes écossais. Plus d'un siècle après sa mort, McTaggart continue d'inspirer les artistes : son influence est particulièrement visible dans l'œuvre de Will Maclean (né en 1941) qui, à la manière de McTaggart, a uni histoire et paysage dans ses monuments érigés dans les Hébrides commémorant l'histoire et la culture des Highlands ${ }^{62}$.

\footnotetext{
century art worldwide, vol. 7, n 1, 2008, page non numérotée. Disponible sur <www.19thc-artworld wide.org/index.php/spring08/111-lochaber-no-more-landscape-emigration-and-the-scottishartist-1849-1895-by-robin-nicholson> [consulté le 10/07/2012].)

61. «Les tableaux de Horatio McCulloch et des paysagistes écossais du XIxe siècle», art. cité, p. 146.

62. Maclean est revenu sur l'influence qu'exercent les tableaux de McTaggart sur son œuvre dans un entretien accordé en 2011 à l'occasion de la rétrospective que lui a consacrée la Fleming Collection, à Londres (disponible sur <http://theahmblog.blogspot.it/2011/03/will-maclean-in-conversationwith-sandy.html> [consulté le 19/07/2012]). Pour les monuments de Maclean, voir L. Gourievidis, "Le présent du passé. La commémoration de l'histoire des crofters sur l'île de Lewis», Études écossaises, n 11, Grenoble, Ellug, 2008, p. 175-192.
} 


\section{Références bibliographiques}

\section{Ouvrages}

Andrews Malcolm, Landscape and Western art, Oxford, Oxford University Press, 1999.

Caw Sir James, William Mc Taggart, Glasgow, James Maclehose and Sons, 1917.

Devine Thomas D., Clanship to Crofters' War. The Transformation of the Scottish Highlands, Manchester et New York, Manchester University Press, 1994.

_- The Scottish Nation 1700-2000, Londres, Penguin Books, 1999.

Duchein Michel, Histoire de l'Écosse, Paris, Fayard, 1998.

Errington Lindsay, William McTaggart, 1825-1910, Édimbourg, National Gallery of Scotland, 1989.

Kvaerne Per, William McTaggart 1835-1910. Singing Songs of the Scottish Heart, Édimbourg, Atelier Books, 2007.

Macdonald Murdo, Scottish Art, Londres, Thames and Hudson, 2000.

Macmillan Duncan, Scottish Art 1460-2000 (1990), Édimbourg et Londres, Mainstream publishing, 2000.

Morrison John, Painting the Nation. Identity and nationalism in Scottish painting, 1800-1920, Édimbourg, Edinburgh University Press, 2003.

Prebble John, The Highland Clearances (1963), Harmondsworth, Penguin Books, 1973.

Roger Alain, Court traité du paysage, Paris, Gallimard, 1998.

Tranter Nigel, The Story of Scotland (1987), Glasgow, Neil Wilson Publishing, 2003.

\section{Articles}

Nicholson Robin, «Lochaber No More - Landscape, emigration and the Scottish artist 1849-1895» [en ligne], Nineteenth century art worldwide, vol. 7, no 1, printemps 2008. Disponible sur <www.19thc-artworldwide. org/index.php/spring08/111-lochaber-no-more-landscape-emigration-and-the-scottish-artist-1849-1895-by-robin-nicholson> [consulté le 10/07/2012].

MorRison John, «Holland and France: prototype and paradigm for nineteenth-century Scottish Art» [en ligne], Fournal of Irish Scottish Studies, vol. 2, n 1, septembre 2008, Aberdeen, Arts and Humanities Research Council Centre for Irish and Scottish Studies, p. 123-138. Disponible sur <www.abdn.ac.uk/riiss/JISS/2.1/2.1_Morrison.pdf> [consulté le 20/06/2012]. 


\section{Tableaux cités}

Allan David, The Fourth Duke of Atholl and Family, 1780, The Blair Charitable Trust, château de Blair.

- The Highland Dance, 1780, National Gallery of Scotland, Édimbourg. Boyd Alice, St Columba's Farewell to the White Horse, v. 1865, œuvre non localisée.

Duncan John, The Awakening of Cuchullin, v. 1895, Ramsay Lodge, Édimbourg.

-, Saint Columba Bidding Farewell to the White Horse, 1925, Andrew Carnegie House, Dunfermline.

FAed Thomas, Oh Why Have I Left My Hame?, 1887, Sunderland Museum and Winter Gardens, Sunderland.

- The Last of the Clan, 1865, Glasgow Museums and Art Gallery, Glasgow.

- Sunday in the Backwoods, 1858, Musée des beaux-arts de Montréal, Montréal.

Grant Sir Francis, Fames Farquharson of Invercauld, 1844, Trustees of the Captain Farquharson's Invercauld Trust.

Henry George et Hornel Edward Atkinson, The Druids: Bringing Home the Mistletoe, 1890, Glasgow Museums, Glasgow.

McTaggart William, Away o'er the Sea - Hope's Whisper, 1889, Kirkcaldy Museum and Art Gallery, Kirkcaldy.

- The Coming of Saint Columba, 1895, National Gallery of Scotland, Édimbourg.

_-, Dora, 1869, Royal Scottish Academy, Édimbourg.

_, The Emigrants - America, 1891-1894, collection particulière.

- The Emigrants Leaving the Hebrides, 1883-1889, Tate Britain, Londres.

—, The Emigrants Leaving the Hebrides, 1891-1898, Kirkcaldy Museum and Art Gallery, Kirkcaldy.

-, The Orange Girl, 1863, œuvre non localisée.

-, The Preaching of Saint Columba, 1895, City of Edinburgh Council, Édimbourg.

—, Puir Weans, 1863, œuvre non localisée.

- The Sailing of the Emigrant Ship, 1895, National Gallery of Scotland, Édimbourg.

—, Spring, 1864, National Gallery of Scotland, Édimbourg.

-, When St Columba Landed, 1904, The Fine Art Society, Londres.

-, Word from the West, 1864, œuvre non localisée.

—, The Wreck of the Hesperus, 1860, James Holloway.

Millais Sir John Everett, Autumn Leaves, 1855-1856, City Art Galleries, Manchester. 
—, The Blind Girl, 1856, Birmingham Museum and Art Gallery, Birmingham.

—, Ophelia, 1851-1852, Tate Britain, Londres.

Nicol John Watson, Lochaber No More, 1883, Fleming-Wyfold Art Foundation, Londres.

ReID Sir George, Autumn, 1876, Aberdeen Art Gallery and Museums, Aberdeen.

-, Montrose, 1888, Aberdeen Art Gallery and Museum Collections, Aberdeen.

Wright John Michael, Sir Mungo Murray, v. 1683, Scottish National Portrait Gallery, Édimbourg. 